Z. Klin. Chem. Klin. Biochem.

12. Jg. 1974 , S. $403-407$

\title{
Enzymatische Bestimmung des Gesamt-Cholesterins im Serum
}

\author{
Von P. Röschlau, E. Bernt und W. Gruber \\ Boehringer Mannheim GmbH, Biochemica Werk Tutzing
}

(Eingegangen am 10. April/13. Juni 1974)

\begin{abstract}
Eine einfache enzymatische Methode zur Bestimmung des Gesamt-Cholesterins im Serum wird beschrieben. Die im Serum vorhandenen Cholcsterinester werden mittels Cholesterinesterase quantitativ in freics Cholestcrin und Fettsäuren gespalten. Das freic Cholesterin wird in Gegenwart von Sauerstoff und Cholesterinoxydase in $\Delta^{4}$-Cholestenon umgewandelt. Das bei dieser Reaktion entstehende Wasserstoffperoxyd oxydiert bei Anwesenheit von Katalase Methanol zu Formaldehyd. Dieses reagiert mit Ammoniumionen und Acetylaceton unter Bildung von 3,5-Diacetyl-1,4-dihydrolutidin, dessen Farbintensität bei $405 \mathrm{~nm}$ gemessen wird. Richtigkeit und Präzision der Methode sind schr gut. Die Proportionalität ist bis 25,85 mmol Cholesterin/l Serum gegeben. Serumprotcinc, Bilirubin, Creatinin, Hämoglobin und Pharmaka stören nicht.
\end{abstract}

\section{Enzymatic determination of total cholesterol in serum}

A simple, enzymatic method for the detcrmination of total cholesterol in scrum is described. All cholesterol esters in the serum are split quantitatively into frec cholesterol and fatty acids by cholesterol esterase. In the presence of oxygen, the free cholesterol is converted by cholesterol oxidase into $\Delta^{4}$-cholestenone. The hydrogen peroxide produced in this reaction oxidizes methanol to formaldehyde in the presence of catalase. The formaldehyde reacts with ammonium ions and acetylacetone to form 3,5-diacetyl-1,4-dihydrolutidinc, which is detcrmined colorimetrically at $405 \mathrm{~nm}$. The accuracy and precision of this method are very good. Proportionality is achieved up to $25.86 \mathrm{mmol}$ cholesterol/l.serum. Serum proteins, bilirubin, creatinine, hemoglobin and drugs do not interferc.

Die Cholesterin-Bestimmung im Serum ist für die klinische Diagnostik von größter Bedeutung, gehört doch die Hypercholesterinämie zu den wesentlichen Risikofaktoren der Arteriosklerose und deren Folgeerscheinungen, jenen Zivilisationskrankheiten, die den ersten Platz unter den Ursạchen für Tod und Invalidität einnehmen $(1-3)$.

Cholesterin kommt im Blut in relativ hoher Konzentration vor. Es ist normalerweise zu etwa $75 \%$ mit Fettsäuren verestert und aufgrund seiner schlechten Wasserlöslichkeit an Lipoproteine gebunden (4). Diagnostisch von Bedeutung ist vor allem die Bestimmung des Gesamt-Cholesterins, d. h., der Summe des freien und veresterten Cholesterins (5).

Die bisher gebräuchlichen Verfahren zưr Bestimmung des Cholesterins sind chemische Methoden basierend auf den Arbeiten von Salkowski (6), Liebermann (7) und Burchard (8), in den Modifikationen nach Zlatkis (9) bzw. Watson (10). Alle diese Methoden sind nicht sp?zifisch, besitzen eine große Fehlerbreite und werden bereits durch geringe Feuchtigkeitsmengen empfindlich gestört (5). So täuschen z. B. $0,17 \mathrm{mmol} / 1$ Bilirubin eine Erhöhung der Cholesterin-Konzentration um etwa 0,78 $\mathrm{mmol} / 1$ Serum vor. Der Hauptnachteil dürfte jedoch in der Verwendung von stark ätzenden Reagenzien, wie konzentrierter Schwefelsäure, Essigsäureanhydrid und Essigsäure, liegen.
Es ist daher nicht verwunderlich, daß auf dem III. Internationalen Symposium über Arteriosklerose 1973 in Berlin, nachdrücklich eine verbesserte Cholesterin-Analytik gefordert wurde. Aufgrund der Erfahrungen bei Glucose und Harnsäure im Rahmen der klinischen Diagnostik, bei denen mit Erfolg unspezifische, störanfällige, chemische Nachweismethoden durch spezifische, enzymatische abgelöst wurden, haben wir versucht, eine enzymatische Cholesterin-Bestimmung zu entwickeln, die möglichst alle Wünsche hinsichtlich Zuverlässigkeit und Handhabung erfüllen sollte. Die halbenzymatische Cholesterin-Bestimmung, d. h., alkalische Verseifung der Cholesterinester mit anschließender Bestimmung des freien Cholesterins mittels Cholesterinoxydase, wurde erstmals von Richmond $(11,12)$. Flegg und Whitehead $(13,14)$ und Röschlau et al. (15) beschrieben. Der Nachteil dieser Methode liegt in der arbeitsaufwendigen und störanfälligen Verseifung (16) sowie bei manchen Methoden in der notwendigen, aber umständlichen Entfernung der bei der alkalischen Hydrolyse entstehenden reduzierenden Substanzen (12).

Wir haben uns daher um die Entwicklung einer Methode zur vollenzymatischen Bestimmung des Gesamt-Cholesterins im Serum bemüht. Die Cholesterinester sollten enzymatisch auf schonende und einfache Weise in freies Cholesterin überführt werden. 


\section{Material und Methoden}

Cholesterinoxydase aus Nocardia erythropolis, Cholesterinesterase aus Mikroorganismen, Katalase (EC 1.11.1.6), Preciset $^{2}$ ), Cholesterin, Precilip ${ }^{2}$ ) sowie die Biochemica Test Combination Cholesterin (chemische Methode), Best.-Nr.: 15949, wurden von Boehringer Mannheim GmbH, die einzelnen Cholesterinester sowie andere Steroid-Derivate von Ega Chemie (Steinheim), Merck (Darmstadt), Serva (Heidelberg), Sigma (St. Louis/USA), Roth (Karlsruhe) und Schering AG (Berlin), Hydroxypolyäthoxydodecan bei Klinke (Hamburg), Cholesterin vom National Bureau of Standards (Washington/USA) bezogen.

Bestimmung der Cholesterinoxydase-Aktivität bei $25^{\circ} \mathrm{C}$

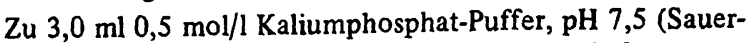
stoff-gesättigt), mit $0,4 \%$ Hydroxy polyäthoxydodecan, werden $0,1 \mathrm{ml}$ Cholesterin-Standard aus Preciset Cholesterin mit $10,34 \mathrm{mmol} / 1$ und $0,02 \mathrm{ml}$ Cholesterinoxydase (etwa $0,5 \mathrm{U} / \mathrm{ml}$ ) gegeben, sofort gemischt, und die Bildung von $\Delta^{4}$-Cholestenon bei $\left.240 \mathrm{~nm}\left(\epsilon=15,5 \mathrm{~cm}^{2} / \mu \mathrm{mol}\right)(15)\right)$ als Extinktionszunahme/ min gemessen. Enzymkonzentration $=10,06 \cdot \Delta \mathrm{E} / \mathrm{min}[\mathrm{U} / \mathrm{ml}$ Probe-Lösungl.

Bestimmung der Cholesterinesterase-Aktivität bei $25^{\circ} \mathrm{C}$

$\mathrm{Zu} 3,0 \mathrm{ml}$ 0,5 mol/1 Kaliumphosphat-Puffer, pH 7,5 mit 0,4\% Hydroxypolyäthoxydodecan, werden $0,05 \mathrm{ml}$ Precilip und $0,02 \mathrm{ml}$ Cholesterinosydase (etwa $50 \mathrm{U} / \mathrm{ml}$ ) gegeben. Nachdem die Oxydation des freien Cholesterin abgelaufen ist, wird mit $0,02 \mathrm{ml}$ Cholesterinesterase-Lösung gestartet, und die Extinktionszunahme/min bei $240 \mathrm{~nm}$ gemessen.

Enzymkonzentration $=9,97 \cdot \Delta \mathrm{E} / \mathrm{Minin}$. [U/ml Probe-Lösung]

Zur Überprüfung der relativen Reaktionsgeschwindigkeiten der verschiedenen Cholesterinester mit Cholesterinesterase wurden jeweils $0,02 \mathrm{ml}$ mit $0,03 \mu \mathrm{mol}$ des jeweiligen Cholesterinesters zu 5,0 ml 0,5 mol/1 Natriumphosphat-Puffer, pH 8,0, der 0,1\% Hydroxypolyäthoxydodecan und $0,1 \mathrm{U}$ Cholesterinoxydase/ Test enthielt, gegeben. Die Reaktion wurde mit 0,2 U Cholesterinesterase gestartet. Die Reaktionszeit bei $37^{\circ} \mathrm{C}$ betrug 6 min; gemessen wurde das gebildete $\Delta^{4}$-Cholestenon bei $240 \mathrm{~nm}$ (15). In dieser Zeit wurde Myristat als bestes Substrat vollständig gespalten.

\section{Vorinkubation von Serum mit Cholesterinesterase}

0,2 ml Humanserum wurden zu 2,0 ml 0,5 mol/1 Kaliumphosphat-Puffer, pH 7,5, mit 0,4\% Hydroxypolyäthoxydodecan, gegeben, mit 0,5-10,0 U Cholesterinesterase versetzt und $20 \mathrm{~h}$ bei Raumtemperatur vorinkubiert.

Halbenzymatische Cholesterin-Bestimmung: siehe 1.c. (15).

Die Cholesterin-Bestimmung nach Liebermann-Burchard und die Triglycerid-Bestimmung wurden mit den Biochemica Test Combinationen von Boehringer Mannheim $\mathrm{GmbH}$ durchgeführt.

Cholesterin-Bestimmung nach Abell: siehe l.c. (4).

Vollenzymatische Cholesterin-Bestimmung

Die für den vollenzymatischen Cholesterin-Farbtest notwendigen Reagenzien ${ }^{1}$ ) werden in 4 verschiedenen Lösungen zusammengefaßt, deren Haltbarkeit bei $+4^{\circ} \mathrm{C}$ mindestens 9 Monate beträgt.

Lösung 1: $\quad 0,6 \mathrm{~mol} / 1$ Ammoniumphosphat-Puffer, $\mathrm{pH}=7$; $1,7 \mathrm{~mol} / \mathrm{l}$ Methanol; $\geqslant 700 \mathrm{U} / \mathrm{ml} \mathrm{Katalase}$

Lösung 2: $\quad 0,42 \mathrm{~mol} / 1$ Acetylaceton; $2,5 \mathrm{~mol} / 1 \mathrm{Methanol}$; $2,1 \%$ Hydroxypolyäthoxydodecan

Lösung 3: $\quad \geqslant 5 \mathrm{U} / \mathrm{ml}$ Cholesterinesterase

Lösung 4: $\quad \geqslant 2,5 \mathrm{U} / \mathrm{ml}$ Cholesterinoxydase

Aus 5 Teilen Lösung 1, 0,25 Teilen Lösung 2 und 0,02 Teilen Lösung 3 wird ein Cholesterin-Reagenz (Lösung 5) hergestellt, das bei $+4^{\circ} \mathrm{C}$ einen Monat, bei $+25^{\circ} \mathrm{C}$ eine Woche haltbar.ist.

\footnotetext{
$\left.{ }^{1}\right)$ Biochemica Test Combination Cholesterin, enzymatischer Farbtest Boehringer Mannheim GmbH
}

Die Messung erfolgt bei einer Wellenlänge von $405-415 \mathrm{~nm}$, z. B. $\mathrm{Hg} \mathbf{4 0 5} \mathrm{nm}$ in Glasküvetten mit einer Schichtdicke von $1 \mathrm{~cm}$; die Inkubationstemperatur ist $37^{\circ} \mathrm{C}$.

Untersuchungsmaterial: Serum oder Plasma.

Pipettierschema:

Auf den Boden von Reagenzgläsern pipettieren:

\begin{tabular}{|c|c|c|}
\hline & Proben-Leerwert & Probe \\
\hline $\begin{array}{l}\text { Probe } \\
\text { Lösung } 5 \text { (Cholesterin-Reagenz) }\end{array}$ & $\begin{array}{l}0,02 \mathrm{ml} \\
5,00 \mathrm{ml}\end{array}$ & $\overline{-}$ \\
\hline \multicolumn{3}{|l|}{ Inhalt der Reagenzgläser gut mischen } \\
\hline $\begin{array}{l}\text { Lösung } 4 \text { (Cholesterinoxydase) } \\
\text { aus dem Reagenzglas des } \\
\text { Proben-Leerwertes abpipettieren }\end{array}$ & - & $\begin{array}{l}0,02 \mathrm{ml} \\
2,50 \mathrm{ml}\end{array}$ \\
\hline \multicolumn{3}{|c|}{$\begin{array}{l}\text { gut mischen, Proben-Leerwert und Probe mindestens } 60 \text { min } \\
\text { bei } 37^{\circ} \mathrm{C} \text { inkubieren. Extinktion der Probe gegen Proben-Leer- } \\
\text { wert messen: E Erobe }\end{array}$} \\
\hline
\end{tabular}

-Die Berechnung der Cholesterin-Konzentration erfolgt über einen mitgeführten Standard (Precimat Cholesterin) mit $5,17 \mathrm{mmol} / \mathrm{l}$.

\section{Ergebnisse und Diskussion}

\section{Cholesterinesterase}

Etwa $75 \%$ des im Serum vorkommenden Cholesterins sind mit Fettsäuren verestert (4). Beaucamp et al. gelang es, aus Mikroorganismen eine Cholesterinesterase zu isolieren (17), die alle im Serum vorkommenden Cholesterinester gemäß Gleichung (1) spaltet (s. Tab. 1).

\section{(1) Cholesterinester $+\mathrm{H}_{2} \mathrm{O}$ Cholesterin- Cholesterin + Fettsäure esterase}

Tab. 1. Relative Reaktionsgeschwindigkeiten der im.Serum vorkommenden Cholesterinester mit Cholesterinesterase

\begin{tabular}{lcc}
\hline Cholesterinester & Vorkommen im Serum & $\begin{array}{l}\text { Relative Reak- } \\
\text { tionsgeschwin- } \\
\text { digkeit bezogen } \\
\text { [\% der Summe] }\end{array}$ \\
\hline Myristat & 1 & 100 \\
Linolat & 43 & 100 \\
Stearat & 3 & 100 \\
Palmitoleat & 6 & 100 \\
Oleat & 24 & 99 \\
Linolenat & 4 & 98 \\
Palmitat & 10 & 93 \\
Arachidat & 1 & 25 \\
Arachidonat & 6 & 7 \\
\hline
\end{tabular}

Die Affinität des Enzyms zu den einzelnen Cholesterinestern ist zwar unterschiedlich, doch werden alle bei geeigneter Wahl der Versuchsbedingungen innerhalb von 15 min gespalten.

Für die quantitative Spaltung der Cholesterinester im Serum sind 0,1 U Cholesterinesterase pro Bestimmung ausreichend. Eine Erhöhung der CholesterinesteraseMenge bis $1 \mathrm{U} / \mathrm{T}$ est oder eine Vorinkubation des Serums 
mit diesem Enzym über 20 Stunden ergaben keine höheren Gesamt-Cholesterinwerte im Serum (s. Tab. 2). Vergleichsuntersuchungen, bei denen die Cholesterinester schonend mit äthanolischer $\mathrm{KOH}$ verseift wurden $(15,18)$, ergaben zur enzymatischen Verseifung übereinstimmende Werte (s. Tab. 2).

Tab. 2. Einfluß der Cholesterinesterasemenge und Vorinkubationsdauer auf die enzymatische Cholesterinbestimmung in verschiedenen Seren. Vergleich zum halbenzymatischen Cholesterintest (Verseifung mit äthanolischer $\mathrm{KOH})$.

\begin{tabular}{|c|c|c|c|c|c|c|c|}
\hline \multirow[t]{2}{*}{ Serum } & \multicolumn{5}{|c|}{$\begin{array}{l}\text { Gesamt-Cholesterin [mmol/1 Serum] } \\
\text { halben- vollenzymatisch } \\
\text { zyma- [U Cholesterinesterasc/Test] }\end{array}$} & & \multirow[t]{2}{*}{$\begin{array}{l}\text { Vorinku- } \\
\text { bations- } \\
\text { zcit [h] }\end{array}$} \\
\hline & & 0,05 & 0,1 & 0,2 & 0,5 & 1,0 & \\
\hline 1 & 4,03 & $\begin{array}{l}3,98 \\
4,11\end{array}$ & $\begin{array}{l}4,01 \\
4,09\end{array}$ & $\begin{array}{l}4,01 \\
4,11\end{array}$ & $\begin{array}{l}4,09 \\
4,16\end{array}$ & $\begin{array}{l}4,0.3 \\
4,09\end{array}$ & $\begin{array}{l}0 \\
20\end{array}$ \\
\hline 2 & 6,78 & $\begin{array}{l}6,72 \\
6,85\end{array}$ & $\begin{array}{l}6,72 \\
6,80\end{array}$ & $\begin{array}{l}6,85 \\
6,85\end{array}$ & $\begin{array}{l}6,88 \\
6,88\end{array}$ & $\begin{array}{l}6,80 \\
6,72\end{array}$ & $\begin{array}{l}0 \\
20\end{array}$ \\
\hline 3 & 8,82 & $\begin{array}{l}8,92 \\
8,84\end{array}$ & $\begin{array}{l}8,90 \\
8,77\end{array}$ & $\begin{array}{l}8,90 \\
8,72\end{array}$ & $\begin{array}{l}8,77 \\
8,92\end{array}$ & $\begin{array}{l}8,82 \\
8,92\end{array}$ & $\begin{array}{l}0 \\
20\end{array}$ \\
\hline
\end{tabular}

\section{Choleste rin oxydase}

Zur Bestimmung des freien Cholesterins isolierten Holz et al. (19) aus Erdbakterien Cholesterinoxydasen, die 1970, als die Arbeiten begannen, in reiner Form noch nicht bekannt waren. Eines dieser Enzyme ist mittlerweile mit einer spezifischen Aktivität von $20 \mathrm{U} / \mathrm{mg}$ im Handel $^{2}$ ). Da neben Cholesterin in vergleichbaren Konzentrationen keine anderen Steroide im Serum vorkommen (20), ist diese Cholesterinoxydase zur enzymatischen Cholesterin-Bestimmung geeignet. Pro Bestimmung sind 0,1 U/Test dieses Enzyms ausreichend (siehe Richtigkeit).

\section{Testprinzip}

Cholesterinoxydase oxydiert Cholesterin unter Sàuerstoffverbrauch zu $\Delta^{4}$-Cholestenon und Wasserstoffperoxid:

Cholesterin $+\mathrm{O}_{2} \frac{\text { Cholesterin- }}{\text { oxydase }} \Delta^{4}$-Cholestenon $+\mathrm{H}_{2} \mathrm{O}_{2}$

Zur Ermittlung des freien Cholesterins empfehlen sich die Messung

a) der Sauerstoffabnahme,

b) des gebildeten $\Delta^{4}$-Cholestenons,

c) des gebildeten Wasserstoffperoxids chemisch, enzymatisch über Peroxydase oder Katalase. Wir haben uns für die Bestimmung des Wasserstoffperoxids mittels Katalase nach folgendem Schema entschieden:

$$
\begin{aligned}
& \text { Cholesterinester }+\mathrm{H}_{2} \mathrm{O} \underset{\text { esterase }}{\stackrel{\text { Cholesterin- }}{\rightleftharpoons}} \text { Cholesterin + Fettsäure } \\
& \text { Cholesterin }+\mathrm{O}_{2} \quad \frac{\text { Cholesterin- }}{\text { oxydase }} \text { Cholestenon }+\mathrm{H}_{2} \mathrm{O}_{2} \\
& \mathrm{H}_{2} \mathrm{O}_{2}+\mathrm{CH}_{3} \mathrm{OH} \quad \text { Katalase }-\mathrm{HCHO}+2 \mathrm{H}_{2} \mathrm{O} \\
& \mathrm{HCHO}+2 \mathrm{CH}_{3} \mathrm{COCH}_{2} \mathrm{COCH}_{3}+\mathrm{NH}_{3} \longrightarrow \mathrm{CH}_{3}
\end{aligned}
$$

Die Farbintensität des gebildeten 3,5-Diacetyl-1,4-dihydrolutidins kann bei 405-4.15 nm gemessen werden.

Dieses Indikatorsystem ist für die enzymatische Bestimmung der Harnsäure verwendet worden (21) und hat sich bei Urica-quant ${ }^{3}$ ) bewährt. Es zeichnet sich vor allem dadurch aus, daß

a) es spezifisch ist;

b) die Probe nicht enteiweißt werden braucht;

c) Pharmaka nicht stören (22).

Es sind für den enzymatischen Cholesterin-Farbtest analog dem enzymatischen Harnsäure-Farbtest - pro Bestimmung nur 4 Pipettierungen notwendig. Die Inkubationsdauer beträgt bei $37^{\circ} \mathrm{C}$ eine Stunde.

Die Berechnung kann über einen wäßrigen CholesterinStandard erfolgen. Ein Standard mit in Eisessig gelöstem Cholesterin ist ungeeignet, da innerhalb kurzer Zeit Cholesterinacetat gebildet wird $(18,23)$. Dies ist jedoch ein schlechtes Substrat der Cholesterinesterase.

Da es sich bei dem entstehenden Dihydrolutidin-Derivat um eine definierte Substanz handelt (24), ist es - auch nach den Erfahrungen bei Urica-quant - möglich, die Berechnung über einen Faktor durchzuführen.

\section{Richtigkeit}

Verschiedenen Seren zugesetztes, in A thanol gelöstes Cholesterin wurde mit dem enzymatischen CholesterinFarbtest quantitativ wiedergefunden (s. Tab. 3). Die

\begin{tabular}{|c|c|c|c|}
\hline $\begin{array}{l}\text { Humanserum } \\
\text { Cholesteringehalt } \\
{[\mathrm{mmol} / 1]}\end{array}$ & $\begin{array}{l}\text { Zugesctztes } \\
\text { Cholesterin } \\
{[\mathrm{mmol} / \mathrm{l}]}\end{array}$ & $\begin{array}{l}\text { Gefundenes } \\
\text { Gesamt-Chole- } \\
\text { sterin } \\
\text { [mmol/1] }\end{array}$ & $\begin{array}{l}\text { Wieder- } \\
\text { findung } \\
\\
\quad[\%]\end{array}$ \\
\hline $\begin{array}{r}0,88 \\
0,88 \\
5,79 \\
5,79 \\
11,47 \\
11,47\end{array}$ & $\begin{array}{l}12,93 \\
25,86 \\
12,93 \\
25,86 \\
12,93 \\
25,86\end{array}$ & $\begin{array}{l}13,85 \\
25,58 \\
18,65 \\
31,68 \\
24,30 \\
36,31\end{array}$ & $\begin{array}{r}100,3 \\
99,4 \\
99,5 \\
100,1 \\
99,2 \\
96,1\end{array}$ \\
\hline
\end{tabular}
Richtigkeit der enzymatischen Cholesterin-Bestimmung

Tab. 3. Wiederfindung von Cholesterin in Humanseren mit dem vollenzymatischen Cholesterin-Farbtest

3) Boehringer Mannhcim GmbH

2) Boehringer Mannheim GmbH 
Tab. 4. Einfluß der Triglyceridkonzentration auf den vollenzymatischen Cholesterin-Farbtest

\begin{tabular}{llrrr}
\hline & $\begin{array}{l}\text { Human- } \\
\text { serum }\end{array}$ & $\begin{array}{l}\text { zugesetzte } \\
\text { Menge Cho- } \\
\text { lesterin } \\
\text { [mmol/l] }\end{array}$ & $\begin{array}{l}\text { gefundene } \\
\text { Menge Ge- } \\
\text { samt-Chol- } \\
\text { esterin } \\
\text { [mmol/1] }\end{array}$ & $\begin{array}{l}\text { Wieder- } \\
\text { findung } \\
\text { [mm }\end{array}$ \\
[mmol/1] & $\begin{array}{l}\text { Cholesterin } \\
\text { [mmol/1] }\end{array}$ & & & \\
\hline 1,44 & 3,36 & 5,17 & 8,56 & 100,6 \\
1,44 & 3,36 & 16,81 & 20,12 & 99,7 \\
3,95 & 3,41 & 3,88 & 7,24 & 98,7 \\
3,95 & 3,41 & 18,10 & 21,49 & 99,9 \\
7,91 & 4,27 & 3,88 & 8,20 & 101,3 \\
7,91 & 4,27 & 12,93 & 16,94 & 98,0 \\
\hline
\end{tabular}

wird auch durch einen erhöhten Triglycerid-Gehalt des Serums, wie er bei den verschiedenen Typen der Hyperlipoproteinämien vorliegen kann, nicht beeinflußt (s. Tab. 4). Bilirubin (bis zu 0,34 mmol/l Serum), Creatinin (bis zu 4,42 mmol/1 Serum), Hämoglobin (bis zu $75 \mu \mathrm{mol} / 1$ Serum), sowie Antikoagulantien in den üblichen Konzentrationen stören nicht. Ferner konnte keine Störung des enzymatischen Cholesterin-Farbtestes durch Pharmaka bei in vitro- und in vivo-Versuchen festgestellt werden (Stähler \& Munz, Publikation in Vorbereitung).

Für die Cholesterinester-Spaltung mit Cholesterinesterase haben wir gezeigt (s. Tab. 1), daß alle im Serum vorkommenden Cholesterinester Substrate dieses Enzyms sind und bei Einsatz von 0,1 U Cholesterinesterase pro Bestimmung quantitativ gespalten werden.

Der Vergleich des vollenzymatischen Cholesterin-Tests mit der bisher üblichen Referenzmethode nach $A b e l l$ (4), bei der die Störungen der üblichen chemischen Methoden vermieden werden, ergab die in Abbildung 1 dargestellte Korrelation. Danach werden mit beiden Methoden gut übereinstimmende Werte gefunden.

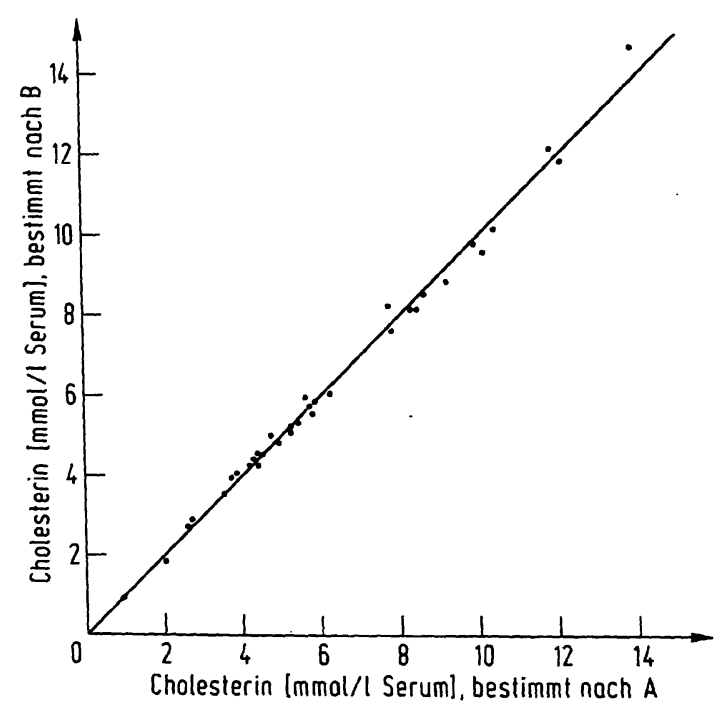

Abb. 1. Korrelation zwischen der Cholesterin-Bestimmung'nach Abell (A) und dem enzymatischen Cholesterin Farbtest (B). Regression: $y=0,999 x-0,01 ; x=0,996$; $s_{y \cdot x}=0,26 ; N=34$.

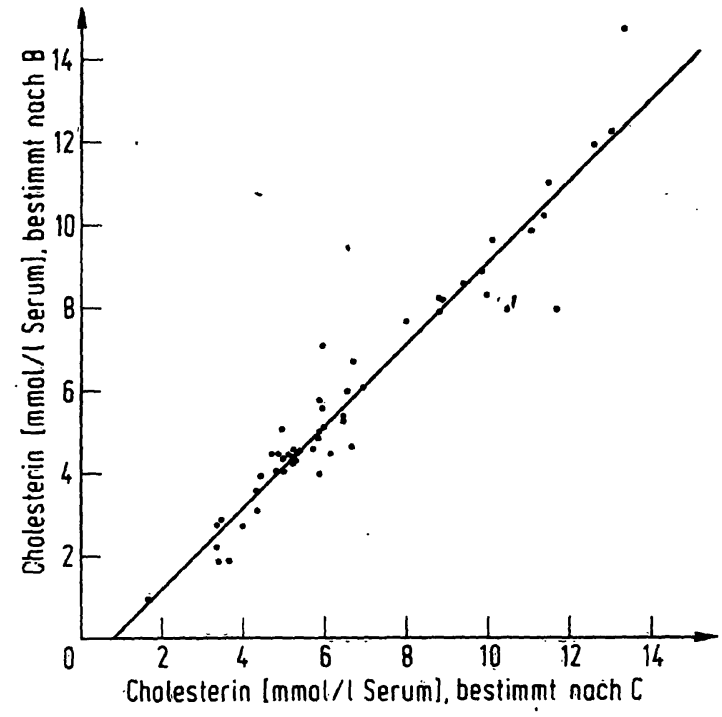

Abb. 2. Korrelation zwischen der Cholesterin-Bestimmung nach Liebermann-Burchard (C) und dem enzymatischen Cholesterin Farbtẹst $(B)$. Regression: $y=0,983 x-0,77$; $\mathrm{I}=0,965 ; \mathrm{s}_{\mathrm{y} \cdot \mathrm{x}}=0,75 ; \mathrm{N}=54$.

Außerdem verglichen wir die chemische Methode nach Liebermann-Burchard $(7,8)$ mit dem enzymatischen Test. Die Regressionsanalyse ergab - wie aus Abbildung $2 \mathrm{zu}$ ersehen ist - ein additives Glied von etwa 0,77 $\mathrm{mmol} / \mathrm{l}$ Cholesterin. Diese Differenz ist $\mathrm{u}$. a. bei der chemischen Methode auf die unspezifische Reaktion von Serum-Bestandteilen, wie z. B. Bilirubin und Serumpròteinen, zurïckzufuihren. Mit dem vollenzymatischen Cholesterin-Farbtest müssen deshalb neue Normalwerte für Cholesterin ermittelt werden.

\section{Präzision}

Der Variationskoeffizient in der Serie liegt bei $2 \%$ $(\mathrm{N}=20 ; 5,17-12,93 \mathrm{mmol} / 1$ Cholesterin), der Variationskoeffizient von Tag zu Tag beträgt etwa $3 \%$ $(\mathrm{N}=20)$.

\section{Eichkurve}

Unter den Testbedingungen besteht direkte Proportionalität zwischen Farbintensität und Cholesterin-Konzentration im Meßbereich 0-25,86 mmol/1 Serum. (s.

Abb. 3).

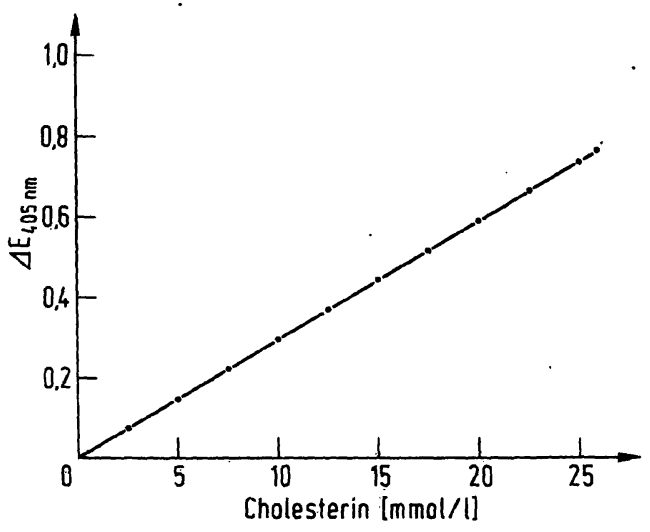

Abb. 3. Eichkurve für den enzymatischen Cholesterin Farbtest. 


\section{Farbstabilität}

Die Farbentwicklung ist nach $60 \mathrm{~min}$ bei $37^{\circ} \mathrm{C}$ abgeschlossen. Danach ist die Färbung für weitere $45 \mathrm{~min}$ stabil.

\section{Empfindlichkeit}

Bei Einsatz von 0,02 $\mathrm{ml}$ Probe/Test entspricht eine Extinktionsdifferenz von 0,153 bei $405 \mathrm{~nm}$ einer Cholesterin-Konzentration von $5,17 \mathrm{mmol} / 1$ Serum. Die Methode ist damit ausreichend empfindlich.

\section{Automaten-Adaptierbarkeit}

Ähnlich wie bei Urica-quant kann dieser enzymatische Cholesterin-Farbtest auf alle diskontinuierlich arbeitenden Automatensysteme übertragen werden (Ziegenhorn, Publikation in Vorbereitung).

\section{Literatur}

1. Lynen, F. (1972), Naturwiss. Rundsch. 25, 382-387.

2. Irsigler, K. (1973), Wien. Klin. Wochenschr. 85, 195.

3. Schwandt, P. (1973), Internist 14, 325-329.

4. Henry, R. J. (1964), Clinical Chemistry, 2. Aufl., S. 843864, Verlag Harper \& Row, New York.

5. Richterich, R. \& Lauber, K. (1962), Klin. Wochenschr. 40, 1252-1256.

6. Salkowski, E. (1872), Pfluegers Arch. 6, 207-212.

7. Liebermann, C. (1885), Ber. 18, 1803-1809.

8. Burchard, H. (1889), Dissertation, Rostock.

9. Zlatkis, A., Zak, B. \& Boyle, A. J. (1953), J. Lab. Clin. Med. 41, 486-492.

10. Watson, D. (1960), Clin. Chim. Acta 5, 637-643.

11. Richmond, W. (1972), Scand. J. Clin. Lab. Invest. 29, Suppl. 126.

12. Richmond, W. (1973), Clin. Chem. 19, 1350-1356.

13. Flegg, H. M. (1973), Ann. Clin. Biochem. 10, 79-84.
Bestimmung des freien und/oder veresterten Cholesterins Führt man die enzymatische Cholesterin-Bestimmung in Abwesenheit der Cholesterinesterase durch, so wird mit Cholesterinoxydase nur das freie Cholesterin erfaßt. Das veresterte Cholesterin ergibt sich dann aus der Differenz von Gesamt- und freiem Cholesterin. Durch getrennte Zugabe von Cholesterinoxydase und Cholesterinesterase ist es natürlich auch möglich, freies und verestertes Cholesterin nacheinander in einem Testansatz zu bestimmen.

\section{Danksagung}

Wir danken Herrn $R$. Herz für seine gewissenhafte Mitarbeit.

14. Whitehead, T. P., persönliche Mitteilung.

15. Röschlau, P., Bernt E. \& Gruber, W. (1974) in Methoden der enzymatischen Analyse (Bergmeyer, H. U., Hrsg.) 3. Aufl., Bd. 2, 1938-1941, Verlag Chemie, Weinheim/ Bergstr.

16. Okey, R. (1930), J. Biol. Chem. 88, 367-369.

17. Beaucamp, K., Lang, G. \& Möllering, H., unveröffentlicht.

18. Röschlau, $P$., unveröffentlicht.

19. Holz, G., Beaucamp, K. \& Lang, G., unveröffentlicht.

20. Zöllner, N. \& Eberhagen, D. (1965), Untersuchung und Bestimmung der Lipase im Blut, Springer-Verlag, Berlin.

21. Kageyama, N. (1971), Clin. Chim. Acta 31, 421-426.

22. Mathies, H., Stähler, F., Wittner, H. \& Vollmar, H. (1974), Med. Klin. 69, 607-612.

23. Klein, B. \& Leinman, N. B. (1974), Clin. Chem. 20, 90-91.

24. Nash, T. (1953), Biochem. J. 55, 416-421.

Dr. P. Röschlau Apoth. E. Bernt Dr. W. Gruber Boehringer Mannheim GmbH Biochemica Werk Tutzing 8132 Tutzing/Obb. 